\title{
STATION CONE ALGORITHM FOR LINEAR PROGRAM WITH NON-NEGATIVE INPUT DATA
}

\section{LE THANH HUE}

Faculty of Informatics Technology

Hanoi University of Mining and Geology

Hanoi

Vietnam

e-mail: lethanhhue@humg.edu.vn

\begin{abstract}
The paper studies the linear programming problem with non-negative input data and applies the station cone algorithm to find the solution. From the nonnegative input data and station cone algorithm's properties, the author has shown how to derive the feasible solution in a simple way. This significantly reduces the computational time, because it skips the feasible solution study phase without having to use the phase I of the simplex algorithm to search. The paper also points out how an interior point is the center of a convex polyhedron in the feasible domain. This is the point in order to connect with the vertex of the station cone which serves to identify the variables in and out of the simplex pivot. Another important part of this paper is the experimental calculation of the station cone algorithm for the above problem class. Tests have shown that the station cone algorithm has fewer iterations than the dual simplex method.
\end{abstract}

2010 Mathematics Subject Classification: 90C05.

Keywords and phrases: linear program, station cone algorithm, dual simplex method.

Received August 11, 2017

(C) 2017 Scientific Advances Publishers 


\section{Introduction}

Derived from the task of supplying supplies to the military, Dantzig has come up with a problem of finding the maximum of a linear function over a convex set [1] - putting the linear programming into one in the greatest mathematical discoveries of the 20 th century.

The practicality of the linear programming up until today, 70 years after Dantzig's work, is still very hot. In all areas of human life, whether in science, technology or economics, in nature or in society, everywhere there is the application of the linear programming. Therefore, any extension or modification of the methods for solving linear programming problems toward better are welcome.

After Khachiyan's fundamental finding on the polynomial time of the linear programming ([2]), the Karmakar algorithm ([3]) became the boom point for speed searches. Thousands of experimental calculations and algorithms have been proposed for linear programming (e.g., [4]). There are several important open problems in the theory of linear programming. One of them is the question: To be or not a variations of simplex algorithm that run in polynomial time [4] ?

In an attempt to improve the algorithmic efficiency of Dantzig simplex method, the authors [5] have introduced the station cone algorithm with impressive computational efficiency. Here in this paper, we apply the station cone algorithm [5] to solve the linear programming with non-negative input data. The main objective of this paper is to carry out experimental calculations for linear programming with non-negative input data and to compare with dual simplex method.

The paper is organized as follows. In Section 2, we introduce the definition of station cone and show how to define a vertex of a station cone for linear programming with non-negative input data. In Section 3, we point out the criteria for the selection of the initial station cone as well as the way of setting up the initial simplex table. The Section 4 
describes how to calculate an interior point which serves to identify the variables in and out of the simplex pivot. The algorithm is presenting in Section 5. The Section 6 presents the computational experiments. Finally, some discussions have been made in Section 7 .

\section{Problem Formulation}

Consider the linear programming problem

$$
\begin{gathered}
\max \sum_{j=1}^{n} c_{j} x_{j}, \\
\sum_{j=1}^{n} a_{i j} x_{j} \leq b_{i}, \quad i=1,2, \ldots, m, \\
x_{j} \geq 0, j=1,2, \ldots, n .
\end{gathered}
$$

Through this paper we suppose that

$$
c_{j}, a_{i j}, b_{i} \geq 0, \quad \forall i, j
$$

Denote $t$ an index from $i=1,2, \ldots, m$, we consider the following problem:

$$
\begin{gathered}
\max \sum_{j=1}^{n} c_{j} x_{j}, \\
\sum_{j=1}^{n} a_{t j} x_{j} \leq b_{t}, \\
x_{j} \geq 0, j=1,2, \ldots, n .
\end{gathered}
$$

Let $V$ be the polytope defined by (2)-(3) and $V_{t}$ be the polytope defined by (6)-(7). Obviously $V \leq V_{t}$. Unlike problem (1)-(3), problem (5)-(7) gives us a much simpler solution. 
Denote $q$ the index so that

$$
\begin{gathered}
\frac{c_{q}}{a_{t q}}=\max _{\forall j \# q} \frac{c_{j}}{a_{t j}}, \\
a_{t q}>0, a_{t j}>0 .
\end{gathered}
$$

Note that, Equation (8) can be achieved in some indices. In such case we choose $q$ as the smallest index. Let $x^{t^{*}}$ be the optimal solution of the problem (5)-(7). We have the following proposition:

Proposition 1. Optimal solution $x^{t^{*}}$ of problem (5)-(7) will be determined by the following formulas:

$$
\begin{gathered}
x_{j}^{t^{*}}=0, \quad \forall j \neq q, j=1,2, \ldots, n, \\
x_{j}^{t^{*}}=\frac{b_{q}}{a_{t q}},
\end{gathered}
$$

where the index $q$ is determined by Equation (8).

Proof. Let $x_{q}^{t^{*}}=x_{j}^{t^{*}}=\alpha, \forall j$, where $\alpha$ is a positive number, it mean $\alpha>0$. From (8), we have:

$$
\frac{\alpha c_{q}}{a_{t q}} \geq \frac{\alpha c_{j}}{a_{t j}}, \forall j=1,2, \ldots, n, j \neq q, a_{t q}>0, a_{t j}>0
$$

Give the value of $\alpha=\frac{b_{q}}{a_{t q}}$ to (10), we have

$$
\frac{b_{q} \cdot c_{q}}{a_{t q} \cdot a_{t q}} \geq \frac{b_{q} c_{j}}{a_{t q} a_{t j}}, \forall j=1,2, \ldots, n, j \neq q, a_{t q}>0, a_{t j}>0
$$

Multiply both sides of (11) with $a_{t q}$, we get

$$
\frac{b_{q} \cdot c_{q}}{a_{t q}} \geq \frac{b_{q} c_{j}}{a_{t q} a_{t j}}, \forall j=1,2, \ldots, n, j \neq q, a_{t q}>0, a_{t j}>0
$$


The formula (12) states that any value $x_{j}>0, \forall j \neq q$ that satisfies $a_{t j} x_{j}<b_{t}$ will have

$$
c_{q} x_{j} \geq c_{j} x_{j}, \quad \forall j \neq q .
$$

The formula (9) asserts that $x^{t^{*}}$ is the feasible solution of (5)-(7) i.e.,

$$
\sum_{j=1}^{n} a_{t j} x_{j}^{t^{*}}=b_{t}, x_{j}^{t^{*}} \geq 0, \quad \forall j=1,2, \ldots, n
$$

Combined with the Equation (13) we get

$$
\sum_{j=1}^{n} c_{t j} x_{j}^{t^{*}} \geq \sum_{j=1}^{n} c_{t j} x_{j} \forall x_{j} \text { so that } \sum_{j=1}^{n} a_{t j} x_{j} \leq b_{t}, x_{j} \geq 0, j=1,2, \ldots, n \text {. }
$$

The proposition has been proved.

Definition of station cone. Polyhedral cone $M$ with a vertex $\bar{x}$ defined by system $\sum a_{i j} x_{j} \leq b_{i}, i=1,2, \ldots, n$ is called the station cone if the vectors $a_{i}, i=1,2, \ldots, n$ are linear independent and if

$$
(c, \bar{x})>(c, x), \quad \forall x \in M .
$$

In the other words, $n$ row linear independent vectors $a_{i}, i=1,2, \ldots, n$ from (2)-(3) will define a station cone if and only if the vector $c$ is a non-negative combination of the vectors $a_{i}, i=1,2, \ldots, n$.

Proposition 2. The optimal solution $x^{t^{*}}$ of problem (5)-(7) which is defined by formulas (8), (9) is a station cone of problem (1)-(3).

Proof. Consider $n$ linear inequalities

$$
\begin{aligned}
& \sum_{j=1}^{n} a_{t j} x_{j} \leq b_{t}, \\
& x_{j} \geq 0, j \neq t .
\end{aligned}
$$


Obviously (14)-(15) define a polyhedral cone $M$ with vertex $x^{t^{*}}$. From the Proposition 1 follows that $x^{t^{*}}$ is an optimal solution of problem (5)-(7). Therefore $\left(c, x^{t^{*}}\right)>(c, x), \forall x \in V_{t}$. On the other side $V_{t} \supset V$ then $\left(c, x^{t^{*}}\right)>(c, x), \forall x \in V$. It means $x^{t^{*}}$ is a vertex of a station cone of problem (1)-(3). The proposition has been proved.

Proposition 3. The points $x^{t^{*}}$ with $t=1,2, \ldots, m$ which is defined by formulas (8)-(9) are the vertices of the station cones of problem (1)-(3).

Proof. The proof derived from Proposition 1 and Proposition 2.

\section{Initial Station Cone and Initial Simplex Table}

Thanks to the above we can find the initial station cone simply.

Let $x^{0}$ be a vertex from $x^{i *}, i \in[1,2, \ldots, m]$, so that

$$
\left(c, x^{0}\right)=\min _{\forall i}\left(c, x^{i^{*}}\right) .
$$

If in (16) there are two or more indicators that the objective function (1) reaches the minimum value, then choose an index of any instance with the smallest value. The station cone with the vertex $x^{0}$ will be the initial station cone in the algorithm in the next section.

As indicated in [5], each vertex of a station cone is a basic solution of the dual problem. Consider the dual problem in matrix form

$$
\begin{gathered}
\min \left(b^{T}, y\right), \\
A^{T} y \geq c^{T},
\end{gathered}
$$

where $c^{T}, A^{T}, b^{T}$ are the transpositions of $c, A, b$. Denote $B$ the basic matrix of $x^{0}$, it means 


$$
B x^{0}=b \text {, }
$$

or

$$
x^{0}=B^{-1} b,
$$

where $B^{-1}$ is the inverse matrix of $B$, i.e., $B B^{-1}=E$, and $E$ is unit matrix. The formula (20) defines a basic solution.

In general, the variables of the initial simplex table will be defined as follows:

$$
x_{j}=B^{-1} A_{j}, \quad j=1,2, \ldots, n+m,
$$

where $A_{j}$ is a column vector. So the formula (21) will define the initial simplex pivot.

\section{Initial Interior Point}

It's easy to see that $\bar{x}^{0}=(0, \ldots, 0)$ is a vertex of the feasible polytope of problem (1)-(3). We define the following $n$ points $\bar{x}^{i}(i=1,2, \ldots, n)$ as follows:

$$
\bar{x}_{j}^{k}=0, \forall j \neq k, \bar{x}_{k}^{k}=\min _{i}\left(\frac{b_{i}}{a_{i k}}\right), a_{i k} \neq 0 .
$$

Calculate

$$
\tilde{x}=\frac{1}{n+1}\left(\bar{x}^{0}+\sum_{k=1}^{n} \bar{x}^{k}\right) .
$$

Obviously $\tilde{x}$ generated by formula (22)-(23) is the barycenter of the polytope with vertices $\left(\bar{x}^{0}, \bar{x}^{1}, \ldots, \bar{x}^{n}\right)$.

Proposition 4. The point is a strict interior point of the feasible region of problem (1)-(3), i.e., 


$$
\begin{gathered}
\sum_{j=1}^{n} a_{i j} \tilde{x}_{j}<b_{j}, \quad i=[1,2, \ldots, m] \\
\tilde{x}_{j}>0, \quad j=1,2, \ldots, n .
\end{gathered}
$$

Proof. From (22) follows that for any coordinate $j$, we have

$$
\sum_{k=1}^{n} \bar{x}_{j}^{k}>0
$$

Combine (23)-(24) we have the following result:

$$
\tilde{x}_{j}>0, \quad j=1,2, \ldots, n .
$$

It means $\tilde{x}_{j}$ satisfies the condition (25) of the proposition. The proposition has been proved.

Now we have to prove that $\tilde{x}$ satisfies condition (24). Indeed, formula (23) confirms that $\tilde{x}$ is the barycenter of the polytope with vertices $\left(\bar{x}^{0}, \bar{x}^{1}, \ldots, \bar{x}^{n}\right)$, therefore

$$
\tilde{x}_{j}=\frac{1}{n+1} \bar{x}_{j}^{j}<\bar{x}_{j}^{j}, \quad \forall j=1,2, \ldots, n .
$$

Combine (22) and (27) we have

$$
\sum_{j=1}^{n} a_{i j} \tilde{x}_{j}<b_{j}, \quad i=1,2, \ldots, m .
$$

This is condition (24) that needs proving.

\section{Algorithm}

We present the algorithm to solve problem (1)-(3) as follows:

Initialization: Determine the initial station cone $M^{0}$ with vertex $x^{0}$ to be the starting cone as in Section 3, satisfying the condition (16) that $\left(c, x^{0}\right)=\min _{i}\left(c, x^{i^{*}}\right)$. 
Determine the innitial simplex table (as in Section 3) according to formulas (20) and (21).

Define an interior point $\tilde{x}$ (as in Section 4) according to formulas (22) and (23).

Set $k=0$ and move to step $k$.

Step $k=(0,1, \ldots)$

1. If $x^{k}$ satisfies all constraints then $x^{k}$ is an optimal solution and stop. If not, connect $\tilde{x}$ with $x^{k}$ to determine the leaving variable and the entering variable.

2. Calculate the new simplex table and go to step $k=k+1$.

Theorem ([5]). The above algorithm produces an optimal solution after a finite number of iterations. 


\section{Computational Results}

The aboved mentioned algorithm has been tested for problems (1)-(3). Input data is randomly generated. The test results are compared with the results of dual simplex algorithm. Followings are the test results.

\begin{tabular}{|c|c|c|c|c|c|}
\hline \multirow{2}{*}{$\begin{array}{c}\text { Variables } \\
n\end{array}$} & \multirow{2}{*}{$\begin{array}{c}\text { Constraints } \\
m\end{array}$} & \multirow{2}{*}{$\begin{array}{c}\text { Math } \\
\text { Problems }\end{array}$} & \multicolumn{2}{|c|}{ Iterations } & \multirow{2}{*}{ Ratio } \\
\hline & & & Dual simplex & Station cone & \\
\hline \multirow{5}{*}{100} & \multirow{5}{*}{200} & 1 & 5143 & 532 & 9.6 \\
\hline & & 2 & 6257 & 685 & 9.1 \\
\hline & & 3 & 5546 & 476 & 11.6 \\
\hline & & 4 & 6325 & 491 & 12.8 \\
\hline & & 5 & 5489 & 402 & 13.6 \\
\hline \multirow{5}{*}{300} & \multirow{5}{*}{1000} & 1 & 197365 & 6318 & 31.2 \\
\hline & & 2 & 214687 & 7126 & 30.1 \\
\hline & & 3 & 185901 & 6549 & 28.3 \\
\hline & & 4 & 177864 & 5835 & 30.4 \\
\hline & & 5 & 224306 & 6974 & 32.1 \\
\hline \multirow{5}{*}{400} & \multirow{5}{*}{1000} & 1 & 381466 & 11258 & 33.9 \\
\hline & & 2 & 350293 & 10762 & 32.5 \\
\hline & & 3 & 341861 & 9584 & 35.6 \\
\hline & & 4 & 323917 & 9673 & 33.4 \\
\hline & & 5 & 360198 & 10249 & 35.1 \\
\hline \multirow{5}{*}{500} & \multirow{5}{*}{1000} & 1 & 570836 & 14214 & 40.1 \\
\hline & & 2 & 556411 & 14432 & 38.5 \\
\hline & & 3 & 532147 & 14540 & 36.5 \\
\hline & & 4 & 516283 & 13917 & 37.09 \\
\hline & & 5 & 524978 & 13368 & 39.2 \\
\hline
\end{tabular}

The test results show that as the number of variables and constraints increases, the station cone algorithm requires fewer iterations than dual simplex algorithm.

Testing computation was conducted on a Macbook laptop; the input matrix was randomly but fully generated so it took a lot of time. The 
computational time would be much less if the test was run on computers with strong configuration and if the inputs matrix was sparse.

\section{Conclusion}

(1) Thanks to the simple calculation of the initial station cone the computational time is reduced.

(2) Testing results show that station cone algorithm has substantially fewer iterations that dual simplex algorithm. The more variables and constraints there are, the more advantage the station cone algorithm possesses.

(3) Testing computation of algorithms requires much time and efforts. The efficiency of the algorithm is of extreme importance to real-life application. In the future, the station cone algorithm shall be tested with larger numbers of variables and constraints, including among others sparse but large matrix.

\section{References}

[1] G. B. Dantzig, Maximization of a linear function of variables subject to linear inequalities, In T. C. Koopmans, Editor, Activity Analysis of Production and Allocation, Wiley, New York (1951), 339-347.

[2] L. G. Khachiyan, A polynomial algorithm in linear programming (in Russian), Doklady Akademiia Nauk SSSR 224 (1979), 1093-1096; English translation: Soviet Mathematics Doklady 20, 191-194.

[3] N. K. Karmarkar, A new polynomial-time algorithm for linear programming, Combinatorica 4 (1984), 373-395.

[4] M. J. Todd, The many facets of linear programming, Mathematical Programming 91 (2002), 417-436.

[5] N. N. Chu, P. C. Duong and L. T. Hue, A new method combining interior and exterior approaches for linear programming, Journal of Mathematics and System Science 5 (ISSN 2159-5291, USA, 2015), 196-206. 